\title{
Molekulare Medizin und medizinische Behandlung kranker Menschen
}

U $\mathrm{m}$ gleich von vornherein Fehlinterpretationen der hier dargestellten Ideen und Vorstellungen zu vermeiden: Ich möchte keinen Zweifel an der eigenen Überzeugung und persönlichen Wertung aufkommen lassen, dass die Methoden und Ergebnisse der so genannten Molekularbiologie einen immensen Zuwachs an Daten und Wissen über den Aufbau und die Funktionen biologischer Systeme im Allgemeinen und insbesondere die des menschlichen Körpers gebracht haben. Bei der Überflutung mit neuen Details ist es allerdings notwendig, den Zusammenhang und die Auswirkungen auf die persönliche fachliche Position zu analysieren, um damit das Ziel des ärztlichen Berufes nicht aus dem Auge zu verlieren - nämlich dem befindlichkeitsgestörten Patienten zu helfen, tatsächliche oder auch vermeintliche Krankheiten zu erkennen und zu behandeln.

Vor diesem allgemeinen Hintergrund ist leicht einzusehen und zu erkennen, dass das Arbeitsfeld der ärztlichen Tätigkeit Bereiche umfasst, in denen weder die moderne molekulare Betrachtungsweise noch deren Methoden geeignet sind, positiv zur Problemlösung beizutragen.

An zwei Bereichen möchte ich erläutern, worin das Dilemma liegt, den Spagat zwischen den sehr attraktiven, leistungsfähigen und schnellen Arbeitsweisen der molekularen Medizin und den eher mühsamen physikalischen und chemischen sowie häufig wenig präzisen Handlungs- und Verständnisebenen der traditionellen organ- und körperbezogenen Medizin bei der Behandlung echt oder scheinbar kranker Menschen immer wieder vollziehen zu müssen. Diese Schwierigkeit überträgt sich, wie sich zeigen lässt, in fataler Weise auf die Neudefinition von Inhalten der Aus-, Fort- und Weiterbildung. Denn diese Hausaufgaben stehen uns immer noch bevor, wenn es um eine wirklich bewertete Neuordnung medizinischer Fachinhalte gehen soll.

So hilfreich bereits heute die molekulare Medizin in genomisch fassbaren Störungen wie den angeborenen Defekten ist: Ein einseitig in diesem Bereich ausgebildeter Arzt wird vor den meisten Herausforderungen wie bei fast allen Akuterkrankungen scheitern - angefangen von der vitalen Gefährdung eines Unfallopfers mit der primären Handlungsmaxime der Lebenserhaltung bis zu den komplexeren Erscheinungsbildern erworbener exogener oder endogener Schädigungen, die von Intoxi- kationen bis zur beispielsweise akuten Unterbrechung der zellulären Blutversorgung reichen. In diesen Bereichen wird die hohe Leistungsfähigkeit der modernen ärztlichen Tätigkeit nur erhalten bleiben, wenn auch in zukünftigen Ausbildungsplänen diesen traditionellen Fertigkeiten eine hohe Priorität eingeräumt wird.

Ein zweiter Bereich zeigt ein weiteres Risiko auf, nämlich die Einschränkung der Fähigkeit zu einer interdisziplinären Betrachtungsweise eines Problems. Nimmt doch die Fähigkeit, Zusammenhänge zu erkennen, in dem Maße ab, in dem die methodische Schulung dieser Betrachtungsweise durch die Zunahme spezifischer zellbiologischer Methoden eingeschränkt wird.

Der klinikarzt verfolgt als eine seiner wesentlichen Zielsetzungen die Absicht, den Themenschwerpunkten in aktualisierten und komprimierten Beiträgen dem komplexen und exponentiell rasant gewachsenen heterogenen Wissen vermittelnd gerecht zu werden.

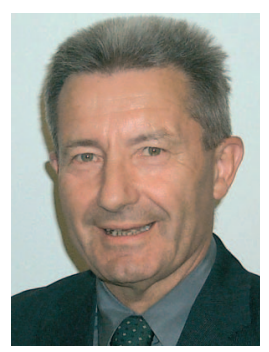

Prof. Dr. Dr. A. Grünert, Ulm

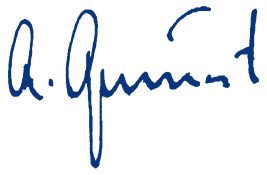

